\title{
Le regard des historiens de la Résistance sur l'engagement des cheminots (1944-1997)
}

Coralie Immelé

\section{OpenEdition}

\section{Journals}

Édition électronique

URL : https://journals.openedition.org/rhcf/532

DOI : 10.4000/rhcf.532

\section{Éditeur}

Rails \& histoire

\section{Édition imprimée}

Date de publication : 1 juin 2006

Pagination : $39-49$

ISSN : 0996-9403

Référence électronique

Coralie Immelé, « Le regard des historiens de la Résistance sur l'engagement des cheminots (1944-1997)», Revue d'histoire des chemins de fer [En ligne], 34 | 2006, mis en ligne le 11 mai 2011 consulté le 22 avril 2022. URL : http://journals.openedition.org/rhcf/532 ; DOI : https://doi.org/ $10.4000 /$ rhcf.532 


\section{Le regard des historiens de la Résistance sur I'engagement des cheminots (1944-1997)}

\section{Coralie Immelé}

Doctorante en histoire, université Lumière - Lyon 2

Les cheminots sont en bonne place dans le panthéon de la mémoire résistante. Nombreux sont, par exemple, les anciens résistants qui, quelle que soit leur appartenance politique, évoquent, dans leurs souvenirs ${ }^{1}$, le rôle qu'ils ont joué durant la clandestinité. Les cheminots apparaissent aussi, épisodiquement, dans la plupart des ouvrages rédigés par les historiens de la Résistance. Mais, au fil du temps, leur regard a profondément évolué. L'histoire de la Résistance, longtemps marquée par les exigences commémoratives, est devenue, à partir des années 1970, fortement problématisée. L'historiographie de la Résistance ${ }^{2}$ a, en effet, connu plusieurs périodes constitutives ${ }^{3}$ avec des intersections et interférences entre les unes et les autres que nous simplifions pour la clarté de l'exposé. De 1944 au milieu des années 1970, l'histoire de la Résistance est écrite exclusivement par ses acteurs. Certains se muent en historiens au sein du tout puissant Comité d'histoire de la Deuxième Guerre mondiale (CHDGM), créé en 1951, qui régit pendant plus de

1- Pierre GUILlain de BÉNOUVILle, Le Sacrifice du matin, Paris, Robert Laffont, 1946, p. 229-230 ; Albert OUZOULIAS, Les Bataillons de la jeunesse, Paris, Éditions sociales, 1967, p. 124, 142, 161, 200, 208-209, 217-219, 315-316, 401, 419 ; Pierre SUDREAU, Au-delà de toutes les frontières, Paris, Odile Jacob, 1991, p. 50-55.

2- Jean-Pierre AZÉMA et François BÉDARIDA, «L'historisation de la Résistance », Esprit, $n^{\circ} 198$ (janvier 1994), p. 19-35 ; Jean-Marie GUILLON, «La Résistance, cinquante ans et deux mille titres après ", in Jean-Marie GUILLON et Pierre LABORIE (dir.), Mémoire et histoire : la Résistance, Toulouse, Privat, 1995, p. 27-43 ; Laurent DOUZOU, La Résistance française : une histoire périlleuse, Paris, Éditions du Seuil, 2005.

3- Nous reprenons les césures thématiques et chronologiques de l'historiographie de la Résistance ("Les témoins gardent la parole et prennent la plume (1944-1974) », « Clio au travail (1944-1978) », «Une historiographie remise en cause et renouvelée (19782002) ») mises en exergue en 2005 par Laurent Douzou, op. cit., p. 83-244. Plus élargies que celles proposées en 1994 par Jean-Pierre Azéma et François Bédarida (1944-1947, 1947-1958, 1959-1969, 1970-1979, 1980-1990) et en 1995 par Jean-Marie Guillon (1944-1951, 1952-1957, 1958-1963, 1964-1975, 1975-1984, 1985-1995), elles semblent ici s'appliquer mieux à l'étude du regard jeté sporadiquement par les historiens de la Résistance sur les cheminots. 
vingt-cinq ans tous les travaux sur l'histoire de la période 1939-1945. Avec la création, en 1978, de l'Institut d'histoire du temps présent (IHTP) qui entraine de fait la disparition du comité, l'histoire de la Résistance entre dans une autre ère. Elle est dominée principalement par le renouvellement de la génération historienne qui, tout en étant nolens volens héritière des travaux de ses ainés, élabore de nouvelles approches conceptuelles.

Notre objectif est ici d'analyser les regards qui ont été portés par ces deux générations d'historiens sur les cheminots et les modes d'écriture qu'ils ont utilisés pour décrire leur engagement. La première, celle des acteurs-historiens, en recensant en arrière-plan les diverses actions menées par les cheminots - individuellement ou collectivement -, contribue in fine à véhiculer, dans la mémoire sociale, l'image de cheminots qui ont beaucoup résisté. L'ouvrage de Paul Durand ${ }^{4}$ grave durablement, dans le marbre, cette représentation sacro-sainte. La seconde génération, celle des historiens qui entrent en scène dans les années 1970, propose de nouvelles grilles de lecture (sociologie de la Résistance ${ }^{5}$ et, en 1997, de nouveaux concepts (intentionnalité et fonctionnalitée), permettant ainsi une meilleure lisibilité de l'engagement des cheminots dans la Résistance et d'en dégager des éléments de compréhension. Ce sont précisément ces deux étapes de l'évolution de l'historiographie de la Résistance que nous allons successivement présenter.

\section{Une description sommaire de la résistance des cheminots, révélatrice des représentations ancrées dans la mémoire sociale (1944-1978)}

L'historiographie de la Résistance est précoce puisqu'elle est entreprise dans les mois qui suivent la libération de Paris. Sur décision du gouvernement provisoire sont créés, en octobre 1944, la Commission d'histoire de l'occupation et de la libération de la France (CHOLF) et, en juin 1945, le Comité d'histoire de la guerre. En décembre 1951, ils fusionnent dans le Comité d'histoire de la Deuxième Guerre mondiale. Ces organismes sont chargés de trois missions : tout d'abord, rassembler toute la documentation existante, puis engranger les témoignages oraux

4- Paul DURAND, La SNCF dans la guerre, sa résistance à l'occupant, Paris, PUF, coll. «Esprit de la Résistance », 1968.

5- Jacqueline SAINCLIVIER, « Sociologie de la Résistance : quelques aspects méthodologiques et leur application en Ille-et-Vilaine », Revue d'histoire de la Deuxième Guerre mondiale, $\mathrm{n}^{\circ} 117$ (janvier 1980), p. 33-74.

6- François MARCOT, «Pour une sociologie de la Résistance : intentionnalité et fonctionnalité », in Antoine PROST (dir.), La Résistance, une histoire sociale, Paris, Les Éditions de l'atelier/Les Éditions ouvrières, 1997, p. 21-41. 
des anciens résistants et, enfin, publier des études historiques sur la Résistance. Pour devenir un membre actif de ces structures, une condition sine qua non : l'enquêteur doit avoir été lui-même résistant car seuls ceux qui ont résisté sont " porteurs d'une vérité, celle de la "vraie" Résistance à laquelle ils ont appartenu $\gg^{7}$ et sont en mesure de rendre compte de ce qu'a été ce phénomène exceptionnel.

Les correspondants débutent par un travail de prospection et partent, entre autres, à la recherche d'archives sur la résistance des cheminots. Jusqu'à l'entrée de Paul Durand dans le Comité d'histoire au cours du deuxième semestre 1956, les collectes effectuées restent peu fécondes : seuls trois rapports relatifs aux actions des cheminots résistants sont versés à la CHOLF entre 1949 et 1951 et une liste de cheminots résistants du département de l'Aube accompagnée de deux rapports d'activité sont expédiés au Comité d'histoire en avril $1953^{8}$.

Dès les années 1950, les acteurs-historiens éditent les premiers résultats de leurs recherches, sous forme de monographies départementales ou sur les organisations de résistance. S’ils évoquent par intermittence le rôle des cheminots dans la Résistance, le mode d'écriture qu'ils emploient pour le présenter est, dans l'ensemble, plutôt descriptif. Une exception toutefois est l'ouvrage de Françoise Bruneau, qualifié par Henri Michel de «martyrologe par de nombreuses citations de noms de résistants $»^{9}$. Cette critique prend tout son sens lorsque l'auteure prend pour exemple les cheminots :

«Les journaux étaient adressés par ses camarades de Nantes à M. Lelay, chef de gare de Varade. [...] De Bordeaux, nos camarades servent le journal à Bègles, où Alexis Duffeau, employé à la SNCF, et René Bissemier, furent d'excellents diffuseurs ${ }^{10}$. » «À la SNCF, André Aquin et l'ingénieur Huguet [mort en déportation], étaient des observateurs précieux ${ }^{11}$.»

En outre, elle rend un hommage pieux aux cheminots résistants en « rend[ant] grâce aux employés de la SNCF [...] pour la distribution comme pour la réception des journaux clandestins $»^{12}$.

7- Jean-Marie GUILLON, « La Résistance, cinquante ans et deux mille titres après », in Jean-Marie GUILLON et Pierre LABORIE (dir.), op. cit., p. 29.

8- IHTP, Bulletin du CHDGM, $\mathrm{n}^{\circ} 4$ (avril 1953), p. 15.

9- Henri MICHEL, Bibliographie critique de la Résistance, Paris, Institut pédagogique national, p. 68 .

10- Françoise BRUNEAU, Essai historique du mouvement né autour du journal clandestin Résistance, Paris, SEDES, 1951, p. 68-69.

11- Ibid., p. 173.

12- Ibid., p. 65. 
Les autres membres du Comité d'histoire mettent en évidence, sans l'argumenter, comme si cela allait de soi, le nombre important de cheminots dans les organisations de résistance. Henri Michel et Marie Granet constatent qu'ils sont nombreux à Combat tout en avouant ne pas connaitre, hormis les noms de quelques chefs, ceux de la plupart des cheminots résistants ${ }^{13}$ ! De même, Marcel Baudot énonce que « Résistance-Fer put compter sur le secrétaire du syndicat des cheminots d'Évreux, Socrate Percebois, sur le secrétaire du syndicat de Serquigny, sur le chef de gare de Gisors-Boisgeloup, Alexandre Bruder, et sur la plupart des cheminots de ces trois centres ferroviaires. L'inspecteur de la SNCF d'Évreux, Jean Detraves, faisait partie de Cohors-Asturies, réseau de renseignements de Libération-Nord $»^{14}$.

La lecture des seuls passages concernant les cheminots dans les ouvrages des acteurs-historiens ne permet d'obtenir qu'une esquisse des diverses activités exercées par les cheminots au sein des organisations de résistance. Henri Michel et Marie Granet relatent que « dans les trains, les employés et, spécialement les ambulants, dissimul[ent] et achemin[ent] du courrier $»^{15}$. Arthur Calmette précise les informations fournies par deux cheminots agents de renseignements : "Chaverot, ex-secrétaire de la Fédération des Cheminots et Delhomme, secrétaire syndical des Petits Cheminots remplissent des missions en province d'où ils rapportent des indications sur les mouvements de trains, les transports de troupe et le ravitaillement, les expéditions en Allemagne, le moral des cheminots ${ }^{16} »$. Et Marie Granet fait simplement remarquer que «les cheminots du Mouvement [Ceux de la Résistance] sabot[ent] les voies ferrées $»^{17}$.

Les membres du Comité d'histoire présentent donc sommairement les actions clandestines des cheminots, soit en citant les activités exercées par tel cheminot, soit en utilisant le vocable « cheminots » toujours au pluriel si bien que leurs actions restent anonymes. L'accumulation

13- Henri MICHEL et Marie GRANET, Combat, histoire d'un mouvement de résistance de juillet 1940 à 1943, Paris, PUF, coll. «Esprit de la Résistance », 1957, p. 230.

14- Marcel BAUDOT, L'Opinion publique sous l'Occupation, Paris, PUF, coll. «Esprit de la Résistance », 1960, p. 149-150.

15- Henri MICHEL et Marie GRANET, op. cit., p. 172.

16- Arthur CALMETTE, L'Organisation civile et militaire, histoire d'un mowvement de résistance de 1940 à 1946, Paris, PUF, coll. «Esprit de la Résistance», 1961, p. 19-20.

17- Marie GRANET, Ceux de la Résistance (1940-1944), Paris, Éditions de Minuit, 1964, p. 109. 
et la récurrence de ces microexemples d'actions individuelles et collectives dans les ouvrages des acteurs-historiens contribuent à distiller, dans la mémoire sociale, l'image de cheminots tous résistants.

L'ouvrage de Paul Durand, La SNCF pendant la guerre, sa résistance à l'occupant, paru en 1968, dans la prestigieuse collection «Esprit de la Résistance » dirigée par le Comité d'histoire, marque une étape décisive dans le processus de construction mémorielle de la représentation des cheminots résistants. Il parachève l'idée, jusqu'alors diffuse, selon laquelle les cheminots auraient résisté dans leur ensemble. Cet ouvrage a pour origine une commande passée au comité, douze ans plus tôt, par Louis Armand, ancien cheminot résistant et Compagnon de la Libération devenu depuis lors président de la SNCF. Lors de la réunion des membres du comité en juillet 1956, le président de la Commission d'histoire de la Résistance, Daniel Mayer, donne lecture d'une lettre d'Armand dans laquelle il demande la rédaction d'une histoire de la SNCF pendant la Deuxième Guerre mondiale qui comprendrait notamment un chapitre sur la résistance des cheminots ${ }^{18}$. Armand confie cette recherche à Paul Durand, inspecteur général honoraire du contentieux de la SNCF et ancien résistant ${ }^{19}$. Durand commence par amasser un nombre important de documents émanant principalement des services de la SNCF (direction générale, mouvement, installations fixes...) et des correspondants départementaux du comité. Il envoie aussi $417^{20}$ questionnaires écrits à d'anciens cheminots résistants. Cette pratique déroge à l'un des principes généraux de travail du Comité d'histoire qui préconise, sauf exception, la règle de l'entretien oral ${ }^{21}$. L'analyse de toutes ces sources le conduit à consacrer plus de $40 \%$ de son livre à la résistance des cheminots qu'il dépeint, dès l'introduction, en ces termes :

18- Bulletin du CHDGM, $\mathrm{n}^{\circ} 45$ (juillet-août 1956), p. 3-4.

19- Centre des archives historiques de la SNCF, Le Mans (CAH SNCF), 138 LM52. L'itinéraire résistant de Paul Durand reste obscur. La fiche de renseignements remplie par la chancellerie de la Légion d'honneur à l'occasion de la demande de son élévation au grade de commandeur - les grades de chevalier et d'officier ayant été obtenus respectivement en juillet 1923 et en décembre 1939 - ne fait pas mention de son activité clandestine. Outre ses états de service militaire pendant et après la Grande Guerre et les différentes fonctions qu'il a occupées au cours de sa vie professionnelle, ses qualités de haut fonctionnaire sont indiquées sans autre précision : «M. Durand, qui s'est toujours montré un juriste de grande classe, en même temps qu'un fonctionnaire d'une rare distinction, a, dans ses fonctions très délicates et lourdes de responsabilité, rendu, avec un dévouement sans limite, d'éminents services au chemin de fer. »

20- Paul DURAND, op. cit., p. 3.

21- Laurent DOUZOU, op. cit., p. 68-69. 
"Restait la résistance individuelle, celle que de nombreux cheminots entreprirent, dans un sentiment général de patriotisme, pur le plus souvent de toute idée politique. Cette opposition à l'occupant naquit au lendemain même de l'armistice; elle se développa continûment. Le nombre effectif des actes de résistance des cheminots de tous grades atteint plusieurs milliers. Il ne saurait être question de les relater tous, encore que nous ayons cherché à situer le plus grand nombre possible. [...] En aucun cas, il ne s'agit ni de dresser un palmarès général, ni d'établir une hiérarchie du mérite individuel. L'anonymat sera donc la règle. Chaque fois que des noms seront exceptionnellement donnés, les raisons en seront évidentes. Ceux des cheminots qui ne retrouveront pas, en ces pages, de relation de leur action personnelle devront comprendre l'impossibilité matérielle qui fut nôtre [...] Si incomplète que soit, certainement, cette histoire de la SNCF en guerre et de sa résistance à l'occupant, l'espoir subsiste d'avoir dit l'essentiel d'un combat magnifique et d'avoir évalué la dette particulière de la nation envers l'ensemble des cheminots ${ }^{22}$. »

Cet extrait annonce d'emblée la thèse soutenue clairement par son auteur, à savoir que les cheminots ont résisté en grand nombre. Cette représentation est défendue par Louis Armand, dès novembre 1944, lors du premier gala organisé par Résistance-Fer - «la tâche des cheminots patriotes était donc variée et nombreux furent ceux qui y participèrent ${ }^{23}$ - et réitérée, crescendo, dans la plupart de ses discours commémoratifs ultérieurs, comme à Innsbruck, en mars 1946 - «les cheminots furent vite assez nombreux dans les rangs de la Résistance $»^{24}$ et dans ses rares écrits abordant le combat clandestin des cheminots :

"Je voudrais, cependant, souligner particulièrement dans cette préface que l'épopée des résistants actifs du rail s'est intégrée dans un consensus quasi unanime de toute la corporation, forte alors de 400000 agents, consensus où ces actifs trouvèrent, en maintes occasions dramatiques, la même protection que s'ils avaient été sous le couvert d'un immense maquis $!^{25} »$

«On n’a pas assez souligné, à mon sens, le caractère unanime du mouvement d'âme qui fermenta chez les cheminots pendant l'occupation : en aucune circonstance on ne vit, je crois, pareil consensus dans un groupement d'hommes aussi important ${ }^{26}$.»

22- Paul DURAND, op. cit., p. 3-4.

23- CAH SNCF, 20 LM751, discours du 9 novembre 1944 au palais de Chaillot, lors du gala organisé par Résistance-Fer, p. 5.

24- Ibid., discours du 18 avril 1946 à Innsbruck, p. 8.

25- Louis ARMAND in Paul DURAND, op. cit., p. viii.

26- Louis ARMAND, Propos ferroviaires, Paris, Librairie Arthème Fayard, 1970, p. 76. 
Paul Durand se situe donc dans le droit fil des propos tenus par Louis Armand. En outre, en plaçant ce travail sous l'égide du Comité d'histoire et en le faisant publier dans sa collection phare « Esprit de la Résistance ", Louis Armand fait labelliser et pérennise de ce fait les conclusions formulées par Paul Durand sur la résistance des cheminots. Cet ouvrage reste longtemps la seule synthèse de référence sur le sujet $\mathrm{t}^{27}$ et les acteurs-historiens, tel Henri Noguères, s'appuient stricto sensu sur les éléments dégagés en substance par celui-ci :

«Paul Durand, consciencieux historien de "la SNCF pendant la guerre" a résumé ainsi ce que l'on peut dire de la participation des cheminots à cette "bataille du rail" qu'ils ont gagnée tous ensemble: "Que l'ensemble du personnel de tous grades appartenant à la SNCF ait adopté, dès les premiers jours de l'occupation une attitude systématiquement hostile aux armées allemandes est, croyons-nous, un fait collectif positivement établi"28. »

Les publications des membres du Comité d'histoire de la Deuxième Guerre mondiale sont somme toute d'un intérêt assez limité pour l'historien qui prend pour thème la résistance des cheminots car les descriptions faites de leurs actions, quand elles existent, sont d'une portée trop générale. Cependant, elles restent incontournables pour l'historien qui décrypte la mémoire de la résistance cheminote parce qu'elles sont porteuses des différentes représentations des cheminots résistants dans la mémoire sociale.

\section{De nouvelles clés de lecture pour comprendre l'engagement des cheminots dans la Résistance (1978-1997)}

La génération d'historiens qui entre en action dans les années 1970, initialement au sein du Comité d'histoire, va rapidement se démarquer de ses aînés en proposant de nouveaux questionnements et approches conceptuelles de la Résistance. S'amorce alors une rupture épistémologique irréversible dans l'historiographie de la Résistance où l'on passe de la description sommaire à une analyse plus fine, problématisée. Deux types de grilles de lecture sont successivement mis en œuvre. Dès la fin des années 1970, les historiens élaborent les premières sociologies de la Résistance et s'intéressent aux parcours individuels des résistants qui leur permettent de saisir, dans son ensemble, le phénomène de la Résistance, aux contours jusque-là imprécis. Dans

27- Christian BACHELIER, La SNCF sous l'Occupation allemande, 1940-1944, Rapport documentaire, Paris, IHTP-CNRS, 1996.

28- Henri NOGUÈRES, La Vie quotidienne des résistants de l'armistice à la libération (19401945), Paris, Hachette Littérature, 1984, p. 151. 
le milieu des années 1990, ils conçoivent des clés de lecture l'intentionnalité, la fonctionnalité et la disponibilité fonctionnelle - pour tenter d'expliquer l'engagement plus important de certains groupes professionnels dans la Résistance. Force est de constater que les cheminots, encore présents en toile de fond dans les monographies rédigées par cette deuxième génération d'historiens, n'échappent pas à ces mutations historiographiques.

Dans les études sociologiques, les cheminots sont situés en tant que membres d'un groupe professionnel à part entière par rapport à l'ensemble de la population résistante examinée. Dans un article précurseur paru en 1980, Jacqueline Sainclivier évalue, après dépouillement des dossiers de demande d'attribution du titre de combattant volontaire de la Résistance, le poids respectif dans la Résistance en Ille-et-Vilaine de 17 grandes catégories socioprofessionnelles (agriculteurs, ouvriers, commerçants, enseignants, employés du secteur public dont cheminots... $)^{29}$. Elle s'interroge alors sur le « lien qui peut exister entre l'activité résistante et l'exercice de tel ou tel métier »:

«L'étude porte ici sur 563 cas [...] On voit apparaître la prédominance du secteur des métaux et de la SNCF, avec respectivement $18,11 \%$ et $17,22 \%$ de l'échantillon. Ces deux secteurs économiques pouvaient rendre d'appréciables services à la Résistance. [...] [Les employés] des ateliers pouvaient faire trainer les réparations des locomotives par exemple; d'autres pouvaient participer à certains sabotages et surtout leur connaissance des horaires de la circulation [...] était indispensable pour l'organisation des sabotages et des déraillements [...] Dans l'ensemble l'analyse socio-économique révèle et confirme tout à la fois le lien évident existant entre certaines catégories socioéconomiques (SNCF [...]) et la Résistance ${ }^{30}$. »

$\mathrm{Si}$ les historiens arrivent à quantifier globalement le nombre de cheminots dans la Résistance, en revanche ils sont beaucoup moins précis, voire démunis, lorsqu'ils essayent de reconstruire leurs itinéraires clandestins :

«Ces pionniers courageux comme [...] Étienne Dusard (le cheminot FTP de Dôle qui s'est pendu dans sa cellule pour ne pas "parler") et tant d'autres... nous sommes incapables de

29- Un travail similaire a été réalisé par Monique LUIRARD, La Région stéphanoise dans la guerre et dans la paix, 1936-1951, Le Puy, Centre d'études foréziennes, 1980, p. 497. Elle a, de son côté, identifié, 19 catégories socioprofessionnelles, dont une spécifique pour les agents de la SNCF qui représentent $2,08 \%$ de la population résistante répertoriée. 30- Jacqueline SAINCLIVIER, art. cit., p. 49 et 51. 
reconstituer de leur œuvre, de dire ce qui les a jetés alors dans la grande aventure ${ }^{31}$. »

Ce constat est néanmoins à nuancer dès lors qu'il s'agit de cheminots occupant une fonction de commandement :

«Depuis l'été 1940, Marcel Hévin, dessinateur à la SNCF, a entrepris de collecter des renseignements à Nantes [...] Fin 1940, un contact avec Londres est établi. Or, à la suite des manifestations du 11 novembre, il a aussi pris la tête d'un petit groupe de jeunes gens, jetant les bases d'un réseau très actif. Mélangeant toutes les activités clandestines [...] le groupe Hévin s'occupe de faire évader des militaires britanniques [...] Marcel Hévin a été arrêté le 25 avril 1941, car un de ses contacts a eu des liaisons avec les premiers agents gaullistes débarqués en Bretagne. Bien qu'ayant bénéficié d’un non-lieu, Marcel Hévin et deux compagnons sont exécutés comme otages au Mont-Valérien après l'attentat de Nantes [contre le feldkommandant Hotz], le 22 octobre $^{32}$. »

Par ailleurs, les historiens mettent l'accent sur le fait que les cheminots sont un rouage essentiel au bon fonctionnement de la Résistance :

« La forte participation [...] de la SNCF à la Résistance s'explique donc aisément par les multiples services qu'ils pouvaient rendre à celle-ci ${ }^{33} . »$

«Les cheminots, outre les renseignements fournis, sont d'utiles convoyeurs (transport de la presse clandestine, de passagers clandestins), mais aussi des saboteurs ${ }^{34}$. »

Ils expliquent ce rôle utilitaire par les caractéristiques intrinsèques du métier de cheminot:

«Les cheminots [...] jouent un rôle considérable dans les franchissements de tous ordres de la ligne de démarcation. Impérieusement liées aux nécessités stratégiques et économiques du moment, leurs activités professionnelles [...] justifient la fréquence de leurs déplacements et une certaine liberté de mouvement $\mathrm{t}^{35}$. »

31- François MARCOT, La Franche-Comté sous l'occupation, 1940-1944, tome 1, La Résistance dans le Jura, Besançon, Cêtre, 1985, p. 16.

32- Christian BOUGEARD, Histoire de la Résistance en Bretagne, Paris, Éditions J.-P. Gisserot, 1992, p. 24.

33- Jacqueline SAINCLIVIER, La Résistance en Ille-et-Vilaine, 1940-1944, Rennes, Presses universitaires de Rennes, 1993, p. 109.

34 Jacqueline SAINCLIVIER, La Bretagne dans la guerre, 1939-1945, Rennes, Éditions Ouest-France, coll. «Seconde Guerre mondiale », 1994, p. 174.

35- François MARCOT, op. cit., p. 29. 
«Pour certaines catégories de professions telles que les cheminots, les postiers, les employés de mairie, les imprimeurs, il est permis de penser que leur appartenance à la Résistance a souvent été déterminée par leur métier. Plus vite que d'autres, ils ont été sollicités qui pour saboter une voie ferrée, qui pour intercepter du courrier, qui pour fournir des cartes d'alimentation, qui pour imprimer un tract $!^{36} \%$.

Il est intéressant de remarquer que les cheminots sont souvent placés en première position par les historiens quand ils énumèrent les différentes professions impliquées dans la Résistance ${ }^{37}$. On peut dès lors se demander si les représentations des cheminots résistants ancrées dans la mémoire sociale ne sont pas aussi latentes dans la mémoire de ces historiens.

L'idée formulée dès 1980 par Jacqueline Sainclivier puis émise à nouveau par d'autres historiens, selon laquelle il y a un lien entre le métier exercé et les activités clandestines entreprises, va être conceptualisée en 1997 par François Marcot, sous les maittres mots d'intentionnalité et de fonctionnalité, pour expliquer la surreprésentation de certains groupes professionnels dans la Résistance (instituteurs, militaires, postiers, cheminots...). Ces deux principes, complémentaires et étroitement liés, renvoient, d'une part, aux intentions qui motivent des hommes et des femmes à faire de la Résistance et, d'autre part, aux besoins des organisations qui sollicitent les acteurs leur apportant, par la fonction qu'ils occupent, une réelle valeur ajoutée. À ces deux concepts s'ajoute une variante, la disponibilité fonctionnelle, proposée par Laurent Douzou : «Elle peut tenir à l'exercice d'une fonction donnée : cheminots, voyageurs représentants placiers en tout genre, garagistes, cafetiers seront rapidement des piliers de l'activité clandestine, les uns parce qu'ils se déplacent beaucoup sans attirer l'attention, les autres parce qu'ils servent de relais [...] La disponibilité fonctionnelle, ce peut être aussi les compétences et accointances qui résultent d’une pratique militante ou civique ${ }^{38}$.»

36- Dominique VEILLON, Le Franc-Tireur. Un journal clandestin, un mouvement de résistance 1940-1944, Paris, Flammarion, 1977, p. 250.

37- Voir également Alya AGLAN, Mémoires résistantes. Histoire du résean Jade Fit̨roy, 1940-1944, Paris, Éditions du Cerf, coll. «L'histoire à vif», 1994, p. 47.

38- Laurent DOUZOU, «L'entrée en résistance», in Antoine PROST (dir.), op. cit., p. $15-16$. 
Ces concepts innovants vont être appliqués, la même année, à la résistance des cheminots par l'historien du social, spécialiste des cheminots, Christian Chevandier qui conclut au " primat de la fonctionnalité $\rangle^{39}$ :

«Fonctionnalité où professionnalité est gage d'efficacité, telle est en effet la clef de l'engagement des cheminots dans la Résistance ${ }^{40}$. [...] Lorsque les cheminots s'engagèrent, ce fut souvent parce que l'occasion s'en offrait plus aisément aux travailleurs du rail ${ }^{41}$. »

Les nouvelles approches élaborées par les historiens, dès la fin des années 1970, ont permis une appréhension du phénomène global de la Résistance. Ils ont, entre autres, déterminé le positionnement et le rôle général joué par les cheminots au sein des organisations de résistance. Les concepts émergeant dans les années 1990 constituent sans nul doute un solide substrat sur lequel il est indispensable de s'appuyer pour arriver, à terme, à une meilleure compréhension de l'engagement individuel des cheminots dans la Résistance.

Les cheminots ont toujours été présentés par les historiens de la Résistance comme des acteurs de second plan, à l'image de la place qu'ils ont occupée durant la clandestinité. Si les formes d'action utilisées sont, dans l'ensemble, bien identifiées (propagande, renseignement, passage clandestin, sabotage...), il n'en est pas de même pour leurs itinéraires individuels largement méconnus. Même le parcours clandestin de Louis Armand, le "plus illustre des cheminots résistants ${ }^{42}$, reste difficile à reconstituer ${ }^{43}$. Les cheminots font encore partie des anonymes et des « oubliés » ${ }^{44}$ de l'histoire de la Résistance, ce qui peut paraître paradoxal pour un groupe professionnel longtemps perçu, dans la mémoire sociale, comme très résistant.

39- Christian CHEVANDIER, «La résistance des cheminots : le primat de la fonctionnalité plus qu'une réelle spécificité », in Antoine PROST (dir.), op. cit., p. 147-158.

40- Ibid., p. 152.

41- Ibid., p. 158.

42- Pour reprendre l'expression du président de la SNCF André Ségalat dans l'avantpropos de l'ouvrage de Paul DURAND, op. cit., p. xi.

43- Au Bureau Résistance et Seconde Guerre mondiale, il n'y a qu'une fiche nominative sommaire et à la chancellerie de l'ordre de la Libération, son dossier de compagnon ne nous donne que peu d'éléments sur son activité résistante entre l'été 1940 et le printemps 1943.

44- En référence au titre de l'ouvrage d'Anne-Marie BAUER, Les Oubliés et les ignorés, Paris, Mercure de France, 1993. 
